\title{
General practice referral of 'at risk' populations to community leisure services: applying the RE-AIM framework to evaluate the impact of a community-based physical activity programme for inactive adults with long-term conditions
}

\author{
E. L. Bird ${ }^{*}(1)$, M. S. Y. Biddle and J. E. Powell
}

\begin{abstract}
Background: In the UK a high proportion of adults with long-term conditions do not engage in regular physical activity. General practice (GP) referral to community-based physical activity is one strategy that has gained traction in recent years. However, evidence for the real-world effectiveness and translation of such programmes is limited. This study aimed to evaluate the individual and organisational impacts of the 'CLICK into Activity' programme - GP referral of inactive adults living with (or at risk of) long-term conditions to community-based physical activity.

Methods: A mixed methods evaluation using the RE-AIM framework was conducted with data obtained from a range of sources: follow-up questionnaires, qualitative interviews, and programme-related documentation, including programme cost data. Triangulation methods were used to analyse data, with findings synthesised across each dimension of the RE-AIM framework.

Results: A total of 602 individuals were referred to CLICK into Activity physical activity sessions. Of those referred, 326 individuals participated in at least one session; the programme therefore reached $30.2 \%$ of the 1080 recruitment target. A range of individual-, social-, and environmental-level factors contributed to initial physical activity participation. Positive changes over time in physical activity and other outcomes assessed were observed among participants. Programme adoption at GP surgeries was successful, but the GP referral process was not consistently implemented across sites. Physical activity sessions were successfully implemented, with programme deliverers and group-based delivery identified as having an influential effect on programme outcomes. Changes to physical activity session content were made in response to participant feedback. CLICK into Activity cost $£ 175,000$ over 3 years, with an average cost per person attending at least one programme session of $£ 535$.

Conclusions: Despite not reaching its recruitment target, CLICK into Activity was successfully adopted. Positive outcomes were associated with participation, although low 6- and 12-month follow-up response rates limit understanding of longer-term programme effects. Contextual and individual factors, which may facilitate successful implementation with the target population, were identified. Findings highlight strategies to be explored in future development and implementation of GP referral to community-based physical activity programmes targeting inactive adults living with (or at risk of) long-term conditions.
\end{abstract}

Keywords: Physical activity, Referral, Social prescribing, Long-term conditions, Implementation, Evaluation, RE-AIM

* Correspondence: emma.bird@uwe.ac.uk

Centre for Public Health and Wellbeing, University of the West of England,

Frenchay Campus, Bristol BS16 1QY, UK

(c) The Author(s). 2019 Open Access This article is distributed under the terms of the Creative Commons Attribution 4.0 International License (http://creativecommons.org/licenses/by/4.0/), which permits unrestricted use, distribution, and reproduction in any medium, provided you give appropriate credit to the original author(s) and the source, provide a link to the Creative Commons license, and indicate if changes were made. The Creative Commons Public Domain Dedication waiver (http://creativecommons.org/publicdomain/zero/1.0/) applies to the data made available in this article, unless otherwise stated. 


\section{Background}

Physical activity is associated with a range of positive physical and mental health outcomes, including a reduced risk of heart disease, obesity, type 2 diabetes, and improved emotional wellbeing $[1,2]$. However, despite clear evidence demonstrating the benefits associated with regular physical activity, self-report data from England suggest that approximately one fifth of men and one quarter of women are inactive (defined as participation in less than 30 min per week of moderate physical activity, or less than 15 min per week of vigorous physical activity), [3] costing an estimated $£ 7.4$ billion each year [4]. Compared with the general UK population, physical inactivity prevalence is even higher among those living with a long-term condition (such as type 2 diabetes and hypertension) [5]. Effective physical activity promotion strategies are therefore required to reduce unsustainable pressure on local health and social care provision [6, 7].

An increase in long-term conditions, alongside an ageing population, has created pressure on the delivery of services in General Practice (GP). This has led to General Practitioners and commissioners in the UK advocating and developing collaborative working practices with social prescribing services in the community [8]. Social prescribing schemes allow primary health care professionals to refer patients to a non-medical service, such as community-based physical activities, art classes, and nature-based activities, with the aim of improving patients' health and wellbeing $[8,9]$.

The benefits of group-based physical activity for those living with long-term conditions are well established, $[10,11]$ and this has resulted in group-based physical activity becoming one of the main activities of social prescription made through GP referral to leisure facilities in the community [8]. However, despite increased interest in social prescribing via GP referral to physical activities delivered in leisure centres, two recent systematic reviews of UK-based social prescribing revealed limited evidence for its effectiveness, with differences in methodological quality and reporting standards identified [9, 12]. Furthermore, there is little evidence for the realworld effectiveness and translation of group-based physical activity programmes specifically targeted at inactive adults with long-term conditions. The most recent systematic review evidence on this topic combine evidence from active and inactive adults, [13] and healthy and unhealthy populations [14]. As such, effort is required to better understand the individual and contextual factors that may facilitate the successful real-world delivery of such programmes, and to generate evidence on their cost and long-term sustainability $[15,16]$.

Review-level evidence suggests that the implementation of effective and sustainable physical activity programmes requires improved transparency and consistency of reporting, $[13,16]$ and further consideration of programme external validity [16]. One framework for evaluating public health programmes that has gained attention in recent years is the Reach, Effectiveness, Adoption, Implementation and Maintenance (REAIM) framework [17]. RE-AIM is a multi-level framework that aims to measure the effects of complex interventions while also identifying the barriers and facilitators to real-world intervention implementation [18]. It has five dimensions which identify factors influencing internal and external validity: Reach of the intervention for the target population; Effectiveness of the intervention on desired outcomes; Adoption of the intervention at setting and staff levels; Implementation, delivery of intervention as intended and participant adherence; Maintenance of intervention effects over time, at individual and organisational levels [17].

CLICK into Activity was a physical activity programme that aimed to support inactive adults living with at least one of the following conditions: type 2 diabetes, prediabetes (defined as those with higher than normal glucose levels, but not meeting criteria for type 2 diabetes), hypertension, and overweight or obesity, to increase physical activity levels. It is an example of social prescribing, with individuals referred to community-based physical activity by a primary health care professional. The overall aim of this study was to evaluate the individual and organisational impacts of CLICK into Activity.

\section{Methods}

\section{CLICK into activity}

A district council located in a rural part of South West England led the CLICK into Activity programme. It was funded by Sport England for 3 years from September 2015 with matched funding support from local partners. A project management team (including members of the district council, local partners and Sport England) developed the programme, including individual and organisational recruitment targets and programme content. The authors were not involved in the programme development phase; involvement was limited to evaluation of the programme.

Gatekeepers from nine GP surgeries agreed to participate in GP referral to community-based physical activity, and three trained exercise specialists experienced in working with inactive adults were recruited to deliver the physical activity sessions. At the time of programme delivery there was no local provision for GP referral that met the UK's recommendations for promoting physical activity among those with long-term conditions [19].

Initially, patients diagnosed with type 2 diabetes, prediabetes and/or hypertension and registered at a participating GP surgery were referred to community-based 
physical activity through one of two channels: 1) Direct contact with a General Practitioner or health care professional during a routine appointment or, 2) GP surgery mail-out to potentially eligible patients based on medical records. Eligibility to participate in the programme was also determined by physical activity levels; only those classified as 'inactive' (individuals reporting total physical activity of 30 or fewer minutes per week) [20] were invited to attend. Early participant recruitment to the programme was low, and so the eligibility criteria were relaxed in December 2017 to include obese and overweight individuals (with a body mass index (BMI) $>25$ $\mathrm{kg} / \mathrm{m}^{2}$ ).

During an initial consultation with an exercise specialist, eligible participants had the opportunity to discuss suitable physical activity options and were encouraged to attend at least one physical activity session per week, but there was no minimum or maximum number of sessions that each participant could attend during their 12-week enrolment period. The physical activity sessions were delivered by exercise specialists, trained in motivational interviewing, in local leisure centres and other community venues, and offered a range of group-based activities (i.e. sessions attended by two or more individuals) in response to evidence supporting group-based physical activity promotion [11, 12]. Sessions were delivered at various times throughout the week, and included circuit training, walking groups, and adapted sports (e.g. seated volleyball). The project management group used local long-term conditions data held by participating surgeries to set a recruitment target for participants. The project aimed to support 1080 participants to attend and participate in at least one 30-min CLICK into Activity session during the 12-week enrolment period.

A logic model, presenting the programme delivery plan and hypothesised outcomes can be found in Additional file 1.

\section{Study design}

To generate evidence on each dimension of the RE-AIM framework a mixed methods approach was utilised. CLICK into Activity participants provided individuallevel data, while organisational-level data were collected from members of the project steering group and the exercise specialists responsible for delivering the programme. Consistent with methods utilised in previous applications of the RE-AIM framework in the area of physical activity promotion, [21, 22] data were obtained from a range of sources: questionnaires, qualitative interviews, and programme-related documentation (Table 1). Evaluation data were collected between September 2015 and June 2018.

\section{Participant recruitment}

This study was granted ethical approval by the University of the West of England Ethics Committee. All participants were provided with information about the evaluation and gave written informed consent to participate.

\section{CLICK into activity participants}

All adults referred to CLICK into Activity were invited to participate in the evaluation at an initial GP surgery consultation with a trained exercise specialist. Data were collected via self-report questionnaires and objective measures administered at baseline (T0), immediately after the 12-week programme (i.e. 3-month follow-up) (T1), 6-month follow-up (T2) and, 12 months after programme completion (T3). Data collection measures were the same at each time point. An exercise specialist conducted T0 data collection during the initial consultation. Follow-up data collection was conducted by an exercise specialist at participating GP surgeries, where possible, or via telephone appointment at the request of a participant. The follow-up data collection mode was not recorded. Each participant was assigned a unique serial number to ensure participant anonymity and to match responses at each data collection point.

\section{Interview participants}

A range of programme stakeholders, including the CLICK into Activity programme manager, a practising General Practitioner (and member of the project steering group), the exercise specialists responsible for delivery, and CLICK into Activity participants were invited to participate in a 30-min semi-structured telephone interview. Organisational-level stakeholders were invited opportunistically during a project steering group meeting.

Table 1 Overview of data sources and their contribution to RE-AIM dimensions

\begin{tabular}{lllll}
\hline Data source & \multicolumn{2}{l}{ RE-AIM dimension } & & Adoption \\
\cline { 2 - 4 } & Reach & Effectiveness & Implementation \\
\hline Questionnaires & $\checkmark$ & $\checkmark$ & $\checkmark$ \\
Semi-structured interviews & $\checkmark$ & $\checkmark$ & $\checkmark$ \\
Programme-related documentation & $\checkmark$ & $\checkmark$ & $\checkmark$ \\
\hline
\end{tabular}

Reach of the intervention for the target population; Effectiveness of the intervention on desired outcomes; Adoption of the intervention at setting and staff levels; Implementation, delivery of intervention as intended and participant adherence; Maintenance of intervention effects over time, at individual and organisational levels [17] 
Telephone interviews with exercise specialists took place approximately 1 year and 2 years into programme delivery, with the programme manager and General Practitioner participating in an interview approximately 2 years into programme delivery. CLICK into Activity participants that agreed to be contacted for interview during initial consultation were invited to participate, with the first ten available recruited to the qualitative aspect of the evaluation. The objective of conducting interviews with multiple stakeholders throughout programme delivery was to gather evidence that could contribute to all dimensions of the RE-AIM framework.

\section{Measures}

\section{Questionnaires}

Self-reported questionnaires assessed weekly physical activity via the short-form International Physical Activity Questionnaire (IPAQ short), [23] which included time spent engaged in moderate and vigorous physical activity, time spent walking and sitting time. Self-reported participation in sport was assessed using the Single Item Sport England Measure: "During the last 7 days, on how many days did you take part in any sport?" (days per week) followed by "How much time did you usually spend doing sport on one of those days?" (hours/minutes per day). Mental wellbeing was assessed using the validated 14-item Warwick Edinburgh Mental Wellbeing Scale (WEMWBS) [24]. Participants were asked to respond to each item, for example "I've been feeling optimistic about the future", on a 5-point Likert scale from 1 (None of the time) to 5 (All of the time). Participants' socio-demographic characteristics (sex, age, ethnicity, health status, education, annual income and relationship status) were also captured. To calculate BMI, an exercise specialist at each time point objectively measured height and weight. Low muscle strength has been linked to higher risk of premature death, [25] and as such, exercise specialists used a dynamometer to measure participant grip strength at each time point. Participants completing follow-up measures via telephone were unable to provide objective grip strength, and height and weight data. For an overview of participant characteristics, please see Table 2 .

\section{Semi-structured interviews}

Qualitative methods were employed by ELB and MB to elicit in-depth feedback on CLICK into Activity, to better understand its impact on physical activity outcomes and to reflect upon implementation (from GP referral to CLICK into Activity sessions), including positive and negative aspects of the programme. A semi-structured interview schedule was developed to explore each of the dimensions of the RE-AIM framework from the perspective of each stakeholder group (CLICK into Activity participants; exercise specialists; organisational-level stakeholders). For example, initial interviews with CLICK into Activity participants and exercise specialists explored individual and organisational programme effectiveness; secondary interviews, conducted with the same individuals approximately one-year later, provided an opportunity to reflect upon earlier responses and discuss individual and organisational programme maintenance. An example interview schedule used in the first interview with CLICK into Activity participants is presented in Additional file 2.

\section{Programme-related documentation}

Exercise specialists recorded participant attendance for the duration of the programme using a paper-based register. These data were entered into an Excel spreadsheet and were used to assess programme participation (participants that attended at least one CLICK into Activity session) and average attendance. Data on resource use and actual costs incurred between programme initiation (September 2015) and June 2018 were provided by the programme manager and used to populate a resource use checklist adapted from previous studies [26, 27].

\section{Data analyses}

Questionnaire data were entered into IBM SPSS Statistics (v.22.0). IPAQ questionnaire data were cleaned in line with IPAQ guidelines [28]. Descriptive statistics were generated for each outcome of interest at each time point. Linear mixed models for repeated measures over time were used to assess whether CLICK into Activity had an effect on participants' physical activity, mental wellbeing and grip strength outcomes, with fixed effects of time and initial participation in at least one CLICK into Activity session. Models were adjusted for baseline socio-demographic characteristics (age, sex, BMI) and GP surgery. This approach was used to account for repeated measures over time and to prevent listwise deletion due to missing data [29]. Data were found not to be missing completely at random (Little's MCAR test, $p=$ 0.00 ) and subsequent missing value analysis indicated that data were missing at random. Bonferroni adjusted post hoc tests compared outcomes to each time point (baseline, 3-month follow-up, 6-month follow-up and 12-month follow-up) to estimate means and $95 \%$ confidence intervals $(95 \% \mathrm{CI})$ for changes in outcomes.

Qualitative audio data were transcribed verbatim and analysed using NVivo 10 (QSR International). Data were explored using thematic analysis, [30] with the coding process based predominantly on mapping data against each of the RE-AIM dimensions in line with recently published guidance [31]. Analysis aimed to generate a balanced assessment of the programme and the factors 
Table 2 Comparison of participant characteristics at baseline according to programme participation

\begin{tabular}{|c|c|c|c|c|c|c|c|}
\hline \multirow[t]{2}{*}{ Characteristic } & \multicolumn{2}{|c|}{ Total sample $(N=602)$} & \multicolumn{2}{|c|}{ Participants $(N=326)^{a}$} & \multicolumn{2}{|c|}{ Non-participants $(N=276)^{a}$} & \multirow[t]{2}{*}{$p^{b}$} \\
\hline & $N$ & $\%$ & $N$ & $\%$ & $N$ & $\%$ & \\
\hline \multicolumn{8}{|l|}{ Sex } \\
\hline Female & 379 & 63.0 & 204 & 62.6 & 174 & 63.3 & 0.87 \\
\hline \multicolumn{8}{|l|}{ Age } \\
\hline $18-34$ & 17 & 2.8 & 6 & 1.8 & 11 & 4.0 & 0.01 \\
\hline $35-50$ & 42 & 7.0 & 14 & 4.3 & 28 & 10.2 & \\
\hline $51-69$ & 229 & 38.0 & 125 & 38.3 & 104 & 37.8 & \\
\hline 70 or above & 309 & 51.3 & 180 & 55.2 & 128 & 46.5 & \\
\hline \multicolumn{8}{|l|}{ Body Mass Index (BMI) } \\
\hline 25 or under & 52 & 8.6 & 34 & 10.4 & 18 & 6.5 & 0.20 \\
\hline $26-29$ & 134 & 22.3 & 70 & 21.5 & 64 & 23.3 & \\
\hline 30 or above & 369 & 61.3 & 193 & 59.2 & 175 & 63.6 & \\
\hline \multicolumn{8}{|l|}{ Ethnicity } \\
\hline White & 580 & 96.3 & 318 & 97.5 & 261 & 94.9 & 0.84 \\
\hline Mixed ethnic group & 3 & 0.5 & 1 & 0.3 & 0 & 0.0 & \\
\hline Black British & 1 & 0.2 & 1 & 0.3 & 2 & 0.7 & \\
\hline Asian & 2 & 0.3 & 1 & 0.3 & 1 & 0.4 & \\
\hline Asian British & 2 & 0.3 & 1 & 0.3 & 1 & 0.4 & \\
\hline Other & 4 & 0.7 & 1 & 0.3 & 3 & 1.1 & \\
\hline \multicolumn{8}{|l|}{ Education } \\
\hline Degree/degree level qualification & 128 & 21.3 & 76 & 23.3 & 52 & 18.9 & 0.15 \\
\hline A level or equivalent & 50 & 8.3 & 24 & 7.4 & 26 & 9.5 & \\
\hline Professional qualification ${ }^{c}$ & 166 & 27.6 & 77 & 23.6 & 88 & 32.0 & \\
\hline O level passes/GCSE passes or equivalent & 91 & 15.1 & 48 & 14.7 & 43 & 15.6 & \\
\hline CSE/SCE & 32 & 5.3 & 15 & 4.6 & 17 & 6.2 & \\
\hline Other & 18 & 3.0 & 15 & 4.6 & 3 & 1.1 & \\
\hline No qualifications & 108 & 17.9 & 68 & 20.9 & 40 & 14.5 & \\
\hline \multicolumn{8}{|l|}{ Annual household income } \\
\hline Up to $£ 9999$ & 84 & 14.0 & 38 & 11.7 & 46 & 16.7 & 0.63 \\
\hline$£ 10,000-£ 19,999$ & 177 & 29.4 & 112 & 34.4 & 65 & 23.6 & \\
\hline$£ 20,000-£ 29,999$ & 77 & 12.8 & 46 & 14.1 & 31 & 11.3 & \\
\hline$£ 30,000-£ 39,999$ & 41 & 6.8 & 15 & 4.6 & 26 & 9.5 & \\
\hline$£ 40,000-£ 49,999$ & 23 & 3.8 & 12 & 3.7 & 11 & 4.0 & \\
\hline$£ 50,000$ or above & 28 & 4.7 & 11 & 3.4 & 17 & 6.2 & \\
\hline Don't know & 99 & 16.4 & 59 & 18.1 & 40 & 14.5 & \\
\hline Prefer not to say & 65 & 10.8 & 32 & 9.8 & 32 & 11.6 & \\
\hline
\end{tabular}


Table 2 Comparison of participant characteristics at baseline according to programme participation (Continued)

\begin{tabular}{|c|c|c|c|c|c|c|c|}
\hline \multirow[t]{2}{*}{ Characteristic } & \multicolumn{2}{|c|}{ Total sample $(N=602)$} & \multicolumn{2}{|c|}{ Participants $(N=326)^{a}$} & \multicolumn{2}{|c|}{ Non-participants $(N=276)^{a}$} & \multirow[t]{2}{*}{$p^{b}$} \\
\hline & $N$ & $\%$ & $N$ & $\%$ & $N$ & $\%$ & \\
\hline \multicolumn{8}{|l|}{ Relationship status } \\
\hline Single & 54 & 9.0 & 26 & 8.0 & 28 & 10.2 & \multirow[t]{7}{*}{0.33} \\
\hline Have partner but do not live together & 7 & 1.2 & 3 & 0.9 & 4 & 1.5 & \\
\hline Live with partner & 47 & 7.8 & 25 & 7.7 & 22 & 8.0 & \\
\hline Married and live with partner & 340 & 56.5 & 192 & 58.9 & 147 & 53.5 & \\
\hline Married and separated from partner & 21 & 3.5 & 9 & 2.8 & 12 & 4.4 & \\
\hline Divorced & 47 & 7.8 & 21 & 6.4 & 26 & 9.5 & \\
\hline Widowed & 79 & 13.1 & 49 & 15.0 & 30 & 10.9 & \\
\hline
\end{tabular}

aparticipants defined as those who attended at least one CLICK into Activity session during the 12-week programme

${ }^{b}$ Comparison of those who attended at least one CLICK into Activity session during the 12-week programme ('participants') with those who did not ('non-participants')

'Equivalent to Nursing, Midwifery, City and Guilds qualifications

that may have had an impact on the reach, effectiveness, adoption, implementation and potential sustainability of CLICK into Activity. Data saturation was reached when further coding was no longer feasible [32]. To confirm accuracy and interpretation of the data during the coding process and at theme development, findings were discussed and agreed between authors and reported in line with COREQ guidelines (see Additional file 3, [33]).

Programme cost data were categorised according to stage of programme delivery as follows: programme preparation cost estimate; programme delivery cost estimate; and, programme research and development cost estimate, to reflect the actual mainstream costs of the programme in a real-world delivery scenario.

\section{Data synthesis}

Triangulation methods [34] were utilised to explore complementary and concurrently collected quantitative and qualitative data, considering evidence from different perspectives with the aim of developing a comprehensive understanding of the population impact of CLICK into Activity, from GP referral to CLICK into Activity session delivery. Consistent with previously utilised methods, [22] data from all sources were synthesised across each dimension of the REAIM framework. Programme reach, effectiveness and maintenance were assessed quantitatively using selfreport and objectively measured data provided by CLICK into Activity participants. Evidence pertaining to effectiveness and maintenance domains is presented together in response to the quantitative analysis approach undertaken. Semi-structured interview data from a range of programme stakeholders were used to provide insight into factors influencing each dimension of the RE-AIM framework. Programmerelated documentation, including attendance records and cost data, provided evidence for programme reach, adoption, and implementation.

\section{Results}

A total of 621 individuals completed baseline measures, with 602 found to be eligible to participate in the CLICK into Activity programme. Three-month follow-up measures were completed by 186 participants (30.9\%), followed by 80 participants at 6 -months (13.3\%), with 41 participants completing a 12-month follow-up questionnaire $(6.8 \%)$.

Twenty-seven telephone interviews were conducted. One interview was conducted with the CLICK into Activity programme manager, and one with a General Practitioner (and member of the project steering group). All three CLICK into Activity exercise specialists took part in two interviews ( 1 year and 2 years into project delivery). Ten CLICK into Activity participants took part in an interview soon after completion of the 12-week programme, with nine completing a second interview 1year post-CLICK into Activity enrolment. One participant emigrated and was unavailable for a second interview. Eight participants attended at least one physical activity session each week during their 12-week enrolment, one had attended 9 sessions, and one participated in 6 sessions. Results corresponding to each of the REAIM dimensions are presented below.

\section{Reach}

Of those eligible to participate in CLICK into Activity $(N=602)$, the majority were referred to the programme due to diagnosis of pre-diabetes, type 2 diabetes or hypertension $(N=558,92.6 \%)$. Following relaxation of the eligibility criteria in December 2017, 22 participants were referred due to being obese or overweight (3.7\%), and 22 were referred for having a combination of longterm conditions (3.7\%). Table 2 reveals that the majority 
of participants were female $(N=379,63.0 \%)$ and just over half of participants were aged 70 or above $(N=309$, 51.3\%). Most participants identified as being of White ethnic origin $(N=580,96.3 \%)$ and just over one fifth were qualified to degree level (or equivalent) $(N=128$, 21.3\%). Almost one third of participants reported an annual household income of between $£ 10,000$ and $£ 20,000$ $(N=177,29.4 \%)$, and most participants described themselves as being in a relationship $(N=394,65.5 \%)$. More than $80 \%$ of respondents had a baseline BMI within the overweight and obese categories (BMI $\geq 25 \mathrm{~kg} / \mathrm{m}^{2}, N=$ $503,83.6 \%$ ).

A range of communication strategies were employed to reach the target audience, including newspaper advertising, display screens at participating GP surgeries, local community sites (online and print), and flyer distribution by exercise specialists in the local area, all advertising the programme. Despite utilising various communication channels, recruitment was low at the outset of the project. However, approximately 18 months into project delivery GP surgeries agreed to a mail-out to all patients diagnosed with at least one of the long-term conditions targeted by the programme. Recruitment figures were seen to increase following mail-outs, and qualitative interviews suggested that a wide ranging and long-term promotional strategy was essential.

I think continual promotion [is important] because there can be a lot of promotion at the beginning of the project, but then not so much as you get into the project. There hasn't been continual promotion with the practice managers. You can't just do [promotional activities] once and expect it to filter through to everybody. Exercise specialist 3

CLICK into Activity aimed to support 1080 individuals to attend and participate in at least one 30-min physical activity session during the 12-week programme. Of the 602 eligible individuals that provided baseline data individuals that provided baseline data, attendance records revealed that 326 participants attended at least one 30 -min session (30.2\% of 1080 target population). These participants attended nine sessions, on average, during the 12-week programme $(M=8.6, S D=6.0)$. Just over one third of participants attended at least 12 sessions during the 12-week enrolment period $(N=104,32 \%)$. These figures suggest that CLICK into Activity sessions were well-liked by participants in that once they had attended one session they often returned for more. As shown in Table 2 analysis identified no differences in sex, ethnicity, relationship status or baseline BMI between those that attended a CLICK into Activity session and those that did not $(p>0.05)$. There was a significantly higher proportion of those aged 70 or above attending a session compared with those that did not $(55.2 \%$ vs $46.5 \%$, respectively; $p=0.01$ ).

Interviews with stakeholder groups identified a range of barriers and facilitators influencing initial participation in a physical activity session. For example, individual-level factors such as personal motivation to lose weight, concerns regarding potential for exacerbating existing health issues, and the fear of embarrassment, were noted. Wider social- and environmental-level factors, including activity scheduling and social support from family, friends and the community were also identified. Qualitative interviews also highlighted the importance of the physical environment, for example the influence of the rural setting on perceptions of neighbourhood safety and subsequent participation.

In rural Somerset the evening sessions were not successful, and that was down to the target population not feeling confident going out when it was dark. So, when the winter came, and the nights were drawing in people weren't feeling comfortable leaving their house to go to an activity session. Exercise specialist 3

\section{Effectiveness and maintenance}

Table 3 presents descriptive statistics for CLICK into Activity outcomes for each data collection point. Linear mixed models analyses revealed time to be a significant predictor of vigorous physical activity, moderate physical activity, walking, sport, total physical activity, sitting time, mental wellbeing, and grip strength $(p<0.001)$. Time and initial participation interactions were not found to be statistically significant $(p>0.05)$.

As shown in Table 4, follow-up pairwise comparisons using Bonferroni adjustments revealed significant baseline to follow-up improvements in vigorous physical activity, moderate physical activity, walking, sport, total physical activity, sitting time, and mental wellbeing across each time point $(p<0.05)$. There was a significant improvement in grip strength from baseline to 3-month and baseline to 12-month follow-up ( $\mathrm{p}<0.05)$, but this change was not evident at 6-month follow-up $(p=0.15)$. Although measures were taken to reduce the effects of missing follow-up data in our analysis, [29] 6- and 12month follow-up response rates were low and as such, these findings should be treated with caution.

Qualitative interviews with CLICK into Activity participants were consistent with quantitative findings, in that numerous positive changes were identified in participants' outlook and perceptions of their health and wellbeing as a result of being referred to the programme. These included increased mobility, weight loss, reduced symptoms from long-term conditions, increased core 
Table 3 Descriptive statistics: CLICK into Activity outcomes

\begin{tabular}{|c|c|c|c|c|c|c|c|c|c|c|c|c|}
\hline \multirow[t]{2}{*}{ Measure } & \multicolumn{3}{|c|}{ Baseline $(N=602)$} & \multicolumn{3}{|c|}{3 month follow-up $(N=186)$} & \multicolumn{3}{|c|}{6 month follow-up $(N=80)$} & \multicolumn{3}{|c|}{12 month follow-up $(N=41)$} \\
\hline & $\bar{N}$ & Mean & $S D$ & $N$ & Mean & $S D$ & $N$ & Mean & $S D$ & $\bar{N}$ & Mean & SD \\
\hline Vigorous $\mathrm{PA}^{\mathrm{a}}$ & 600 & 0.65 & 4.71 & 184 & 58.59 & 68.28 & 79 & 82.41 & 76.89 & 41 & 53.66 & 80.89 \\
\hline Moderate $\mathrm{PA}^{\mathrm{a}}$ & 599 & 10.11 & 19.88 & 184 & 127.20 & 162.15 & 79 & 82.41 & 76.78 & 41 & 72.32 & 79.28 \\
\hline Walking ${ }^{a}$ & 594 & 39.67 & 60.04 & 185 & 114.76 & 135.58 & 80 & 88.69 & 78.45 & 41 & 86.59 & 78.19 \\
\hline Total PA ${ }^{a, b}$ & 592 & 50.54 & 63.45 & 184 & 300.84 & 283.10 & 79 & 253.10 & 183.34 & 41 & 212.56 & 195.38 \\
\hline Sport $^{a}$ & 598 & 0.20 & 2.31 & 185 & 19.76 & 33.82 & 78 & 21.35 & 43.48 & 41 & 21.83 & 42.60 \\
\hline Sitting time ${ }^{a}$ & 572 & 3092.48 & 1362.15 & 177 & 2296.36 & 985.43 & 75 & 2214.80 & 966.23 & 41 & 2053.90 & 838.05 \\
\hline Mental wellbeing ${ }^{c}$ & 485 & 47.74 & 10.88 & 177 & 53.90 & 9.94 & 76 & 57.25 & 8.46 & 40 & 58.35 & 10.47 \\
\hline Grip strength (Llbs) & 596 & 25.43 & 10.01 & 176 & 26.63 & 9.66 & 71 & 26.43 & 9.04 & 34 & 25.03 & 8.02 \\
\hline
\end{tabular}

Numbers do not always sum up to total due to missing responses

PA physical activity

${ }^{\text {a }}$ Minutes per week

${ }^{\mathrm{b}}$ Sum of physical activity data (vigorous PA, moderate PA and walking)

${ }^{c}$ Total Warwick Edinburgh Mental Wellbeing Scale (WEMWBS) score out of a possible 70 points

d Objectively-measured via dynamometer

strength, and increased purpose and feelings of happiness.

To sum it up in a sentence, it's brought me back to life. It's as if I have been in hibernation. I'm a lot happier, fitter, and I can do that little bit more...I've lost quite a lot of weight, and I am a happier, happier person since. It's given me more hope and a more positive attitude. It just does me so much good. I even bought a t-shirt, believe it or not. It says, 'I got CLICKed into Life'. Participant 3

At the end of the 12-week enrolment period, CLICK into Activity participants were signposted to a range of alternative local physical activity classes. Feedback from qualitative interviews indicated that these were best received when personally recommended by an exercise specialist, as they were seen to provide trusted advice. Data also suggested that participants held positive intentions to continue to participate in physical activity after completing the programme. Participants were asked to discuss their views on paying for a service such as CLICK into Activity in the future. Most participants accepted that for such a programme to be sustained a personal contribution towards running costs was to be expected. However, it was also acknowledged that most attendees were of pensionable age with limited disposable income, so subsidised rates would be welcomed.

At an organisational level, steering group members reflected upon the potential sustainability of the programme, and highlighted concerns about engagement from GP surgeries beyond project funding.

Everyone was very enthusiastic [about the GP referral process] at the beginning [of the project] but it's tailed off and I think this is because of the pressures that the
[GP] surgeries are under. People have stopped thinking about good ideas and prevention but just gone to fire-fighting mode. General Practitioner

Referral to physical activity sessions continued at seven of nine surgeries until the end of project funding, but maintenance of the programme from referral through to activity session delivery beyond this time was uncertain due to the challenging economic climate.

\section{Adoption}

A total of nine GP surgeries adopted CLICK into Activity for at least some of the programme delivery period. Eight surgeries were originally invited to participate in the programme at the outset, but two were withdrawn due to low recruitment rates and low project buy-in; one 2-years into project delivery (November 2017) and the other in June 2018. In June 2017 a ninth GP surgery was invited to participate.

Interviews with members of the project steering group revealed that a surgery's decision to adopt the CLICK into Activity programme was associated with a range of engagement activities with practice staff arranged in advance of programme sign-up and delivery. One example included a talk from an external General Practitioner with a specialism in physical activity; this was thought to highlight the importance of physical activity promotion, and thus increase buy-in at staff and setting levels. Qualitative interviews also identified the importance of the presence of a GP staff member visibly recommending and championing the programme in advance of programme adoption. For example, an interview with the CLICK into Activity project manager revealed that the practice manager of one GP surgery - the surgery that was invited to adopt the project following the withdrawal of two 
Table 4 Effectiveness and Maintenance of CLICK into Activity: results from mixed linear models

\begin{tabular}{|c|c|c|c|c|}
\hline & Estimate (change in minutes/week) & SE & $d f$ & $95 \% \mathrm{Cl}$ \\
\hline \multicolumn{5}{|l|}{ Vigorous PA } \\
\hline Baseline to 3-month follow-up & $58.59^{* *}$ & 3.36 & 655.23 & $50.53,66.66$ \\
\hline Baseline to 6-month follow-up & $62.24^{* *}$ & 6.77 & 729.08 & $45.99,78.49$ \\
\hline Baseline to 12-month follow-up & $55.87^{* *}$ & 6.22 & 692.85 & $40.96,70.79$ \\
\hline \multicolumn{5}{|l|}{ Moderate PA } \\
\hline Baseline to 3-month follow-up & $116.99^{* *}$ & 7.30 & 667.55 & $99.47,134.51$ \\
\hline Baseline to 6-month follow-up & $50.88^{* *}$ & 14.96 & 808.68 & $14.98,86.78$ \\
\hline Baseline to 12-month follow-up & $64.34^{* *}$ & 13.72 & 810.94 & $31.42,97.27$ \\
\hline \multicolumn{5}{|l|}{ Walking } \\
\hline Baseline to 3-month follow-up & $65.73^{* *}$ & 6.51 & 487.88 & $50.08,81.38$ \\
\hline Baseline to 6-month follow-up & $39.42^{*}$ & 14.19 & 694.91 & $5.36,73.47$ \\
\hline Baseline to 12-month follow-up & $37.83^{*}$ & 13.30 & 805.96 & $5.92,69.74$ \\
\hline \multicolumn{5}{|l|}{ Total PA ${ }^{a}$} \\
\hline Baseline to 3-month follow-up & $234.87^{* *}$ & 12.21 & 622.24 & $205.56,264.185$ \\
\hline Baseline to 6-month follow-up & $151.56^{* *}$ & 25.23 & 804.96 & $91.04,212.09$ \\
\hline Baseline to 12-month follow-up & $158.60^{* *}$ & 23.14 & 804.86 & $103.11,214.10$ \\
\hline \multicolumn{5}{|l|}{ Sport } \\
\hline Baseline to 3-month follow-up & $20.04^{* *}$ & 1.72 & 15.92 & $15.92,24.16$ \\
\hline Baseline to 6-month follow-up & $21.28^{* *}$ & 3.66 & 12.50 & $12.50,30.07$ \\
\hline Baseline to 12-month follow-up & $29.54^{* *}$ & 3.38 & 21.44 & $21.44,37.65$ \\
\hline \multicolumn{5}{|l|}{ Sitting time } \\
\hline Baseline to 3-month follow-up & $-726.14^{* *}$ & 91.96 & 248.91 & $-947.78,504.50$ \\
\hline Baseline to 6-month follow-up & $-711.54^{* *}$ & 191.83 & 136.03 & $-1176.52,-246.56$ \\
\hline \multirow[t]{2}{*}{ Baseline to 12-month follow-up } & $-632.73^{* *}$ & 159.71 & 112.72 & $-1020.86,-244.59$ \\
\hline & Estimate (change in score) & SE & $d f$ & $95 \% \mathrm{Cl}$ \\
\hline \multicolumn{5}{|l|}{ Mental wellbeing ${ }^{b}$} \\
\hline Baseline to 3-month follow-up & $5.56^{* *}$ & 0.62 & 223.10 & $4.05,7.06$ \\
\hline Baseline to 6-month follow-up & $6.33^{* *}$ & 1.38 & 244.32 & $2.99,9.66$ \\
\hline Baseline to 12-month follow-up & $9.09^{* *}$ & 1.42 & 142.84 & $5.65,12.53$ \\
\hline \multicolumn{5}{|l|}{ Grip strength (Llbs) ${ }^{c}$} \\
\hline Baseline to 3-month follow-up & $1.59^{* *}$ & 0.38 & 204.99 & $0.69,2.50$ \\
\hline Baseline to 6-month follow-up & 1.58 & 0.80 & 175.44 & $-0.35,3.51$ \\
\hline Baseline to 12-month follow-up & $2.15^{*}$ & 0.76 & 73.89 & $0.29,4.00$ \\
\hline
\end{tabular}

PA physical activity. Models adjusted for age category (18-34 years, 35-50 years, 51-69 years, 70 years and above), sex (male/female), BMI category (25 and under, $26-29,30$ and above), GP surgery, and initial participation in at least one CLICK into Activity session (yes/no)

originally recruited surgeries - had lobbied for involvement with the project from its initiation and was highly enthusiastic about physical activity promotion. The CLICK into Activity project manager felt that without this enthusiasm from one member of the team, the surgery and its staff would not have agreed to adopt the programme.

\section{Implementation}

Exercise specialists reported that a key influence on effective GP referral implementation was the presence of a practice manager or staff member who had an appreciation for the value of physical activity for patients with, or at-risk of a long-term condition. Notably, an assessment of programme documentation found that the late 
joining surgery referred 66 participants in 12 months (11.0\% of total sample), while the two surgeries withdrawn from the project referred only 49 participants between them (8.1\% of total sample). One of the exercise specialists expressed surprise at the attitudes of some health professionals at participating surgeries. It was noted that physical activity was not always seen as a priority prevention strategy, and that this had a negative impact on successful implementation.

I thought 'Great I'm going to be a team with the doctors, we are going to really work together'. I thought doctors would know the benefits of exercise, but I was shocked to see that some of them needed educating. They didn't believe in exercise. Exercise specialist 1

Reflections on physical activity session implementation revealed that exercise specialist characteristics were integral to success. Participants reported that exercise specialists created a safe and supportive environment, instilling confidence in them from initial consultation to the end of the programme. Support and guidance from exercise specialists to participate in appropriate tailored physical activity was perceived to promote increased feelings of control over participants' health and wellbeing. Participants also reported feelings of increased self-worth and happiness because of their engagement with exercise specialists.

The only words to describe [the exercise specialist] are 'excellent' and 'awesome'. She is very, very dedicated. She deserves a medal, literally. She is very, very good; she knows what she's doing. Participant 2

Another important feature of programme implementation success was the group delivery of CLICK into Activity sessions. Programme sessions created an opportunity for social engagement with members of the local community with similar health profiles. Many participants reported feelings of social isolation prior to CLICK into Activity, which improved through meeting new people and building social support through the programme.

I think everybody was a bit nervous to start, and then as you got to know people and more and more people joined, the old [participants] were, like, helping the new [participants]. It was just amazing because everybody said they were so nervous, and the older [participants] made the new [participants] feel so welcome. Participant 3

Qualitative interviews with CLICK into Activity participants also revealed that the content of the physical activity sessions was important, with praise for circuit training activities tailored to individuals' needs and abilities. In response to the popularity of circuit training sessions observed through attendance figures and anecdotal feedback provided to exercise specialists, the CLICK into Activity schedule was adjusted over time, with the provision of adapted sports sessions reduced in the final year of project delivery to allow additional capacity for circuit training.

The type of class was important. We did have adapted sports, like table tennis and then Boccia and 'new age' curling. Some of these are really good fun to attend but I think for this type of project [with inactive adults], the circuit style delivery is better and [it] was much more popular. I think this was because people felt they were getting more for their time. The numbers are picking up where adapted sports has been swapped for circuits. Project manager

[The exercise specialist] knows how to sort of treat us 'older people', in the fact that caution has to be adhered to. You know, you don't want to push people too hard. Participant 10

Interviews revealed that successful implementation of the programme was reliant on good communication across all levels of programme delivery. It was reported that quarterly steering group meetings were not always well attended due to competing pressures on project partners' time, but they were perceived to provide an opportunity to share good practice and draw upon expertise from those working in a different field but working toward the same objective.

As shown in Table 5, the total cost of CLICK into Activity implementation over 3 years was $£ 174,396$ (201718 prices), based on total annual delivery and preparation costs. The average cost per person attending at least one CLICK into Activity session was £535. Annual preparation cost estimates, including training expenses, were relatively consistent ( $£ 3000-£ 4000$ each year), while delivery costs were seen to reduce over time as the programme became more established. Research and infrastructure development costs, including IT infrastructure, and evaluation and research expenses totalled $£ 67$, 500 over 3 years. These costs were excluded from the total cost of implementation, as they would not apply to mainstream implementation.

\section{Discussion}

This study applied the RE-AIM framework to evaluate the individual and organisational impacts of GP referral of inactive adults living with (or at risk of) long-term conditions to community-based physical activity. The 
Table 5 CLICK into Activity costs and resources

\begin{tabular}{|c|c|c|c|}
\hline & $\begin{array}{l}\text { Nov } 2015 \text { - Oct } 2016 \\
£ \text { actual }\end{array}$ & $\begin{array}{l}\text { Nov 2016-Oct } 2017 \\
£ \text { actual }\end{array}$ & $\begin{array}{l}\text { Nov } 2017 \text {-Oct } 2018 \\
£ \text { estimate }\end{array}$ \\
\hline \multicolumn{4}{|l|}{ Delivery Cost Estimate } \\
\hline Staff (Salaries two exercise specialists) & 42,128 & 43,368 & 41,215 \\
\hline Equipment & 4002 & 1723 & 1250 \\
\hline Hire of Facilities & 27,063 & 26,696 & 25,000 \\
\hline Surgery Room Hire (in Kind) & $-22,080$ & $-22,080$ & $-22,080$ \\
\hline Promotion \& Publicity & 7358 & 3210 & 4000 \\
\hline Transport/Travel & 441 & 360 & 500 \\
\hline Sub Total & 58,912 & 53,277 & 49,885 \\
\hline \multicolumn{4}{|l|}{ Preparation Cost Estimate } \\
\hline Training \& Coaching fees/expenses & 4871 & 4451 & 3000 \\
\hline Sub-total & 4871 & 4451 & 3000 \\
\hline \multicolumn{4}{|l|}{ Research \& Infrastructure Development } \\
\hline IT Infrastructure & 22,500 & - & - \\
\hline Evaluation \& Research & 12,500 & 16,250 & 16,250 \\
\hline Sub-total & 35,000 & 16,250 & 16,250 \\
\hline Annual Cost of Implementation (Preparation and Delivery) & 63,783 & 57,728 & 52,885 \\
\hline \multicolumn{3}{|c|}{ Total Cost of Implementation over 3 Years from a funder perspective ${ }^{a}$} & $£ 174,396$ \\
\hline \multicolumn{3}{|c|}{ Average Cost per person based on at least one attendance at CLICK into Activity 12-week programme ${ }^{b}$} & $£ 535$ \\
\hline
\end{tabular}

${ }^{a}$ Sum of delivery cost estimate and preparation cost estimate. Excludes research and infrastructure development costs. ${ }^{\mathrm{b}}$ The average cost per person attending at least one CLICK into Activity session = a / number of people attending at least one session $(N=326)$

collection of quantitative and qualitative data from a range of sources, and the application of the RE-AIM framework, helped to identify the impacts of the programme while also highlighting potential barriers and facilitators to real-world implementation.

CLICK into Activity reached just under one third of the target population (30.2\%). It is difficult to assess this figure against similar studies, as reported estimations of reach often fail to reflect the true number of eligible participants and those that go on to participate in an intervention [13, 16]. Recruitment figures were seen to increase following GP mail-outs, adding to the evidence base in support of utilising active recruitment strategies (e.g. health professional referral or targeted mail-out [35]) to engage a representative target audience for a physical activity programme [36]. Relaxation of the eligibility criteria to include obese and overweight individuals saw recruitment increase to some extent. However, recruitment was most actively impacted by GP mail-out. These findings highlight the importance of taking a flexible approach to marketing and recruitment to promote programme reach. Factors contributing to initial (non)participation were numerous and varied, including individual-, social-, and environmental-level barriers and facilitators. These findings are consistent with those from a qualitative review of reasons for physical activity participation among children and adults [37]. Future programmes may need to consider and address some of the socio-ecological barriers preventing inactive adults from initial attendance, as our findings suggest that once an individual attends one session they often return.

Overall, positive quantitative and qualitative findings were found for programme effectiveness, and many positive outcomes were maintained up to 12 months. Follow-up physical activity responses exceeded UK physical activity recommendations for adults and older adults [38] and are particularly encouraging in light of UK Government targets to tackle inactivity $[6,7]$. No differences were observed in target outcomes between those that attended a physical activity session and those that did not. No qualitative interviews were conducted with individuals that did not attend a session and therefore it is difficult to interpret this finding. However, this finding is consistent with those reported elsewhere [39, 40]. One possible explanation is that the GP referral process was a brief intervention in itself, encouraging individuals to seek out activity independently; this warrants further examination.

Programme adoption at GP surgeries was successful, with all nine surgeries invited taking up the programme. However, there was only partial adoption from two surgeries, which were withdrawn from the project. Consistent with findings from a recent systematic review, [9] our study identified a range of factors influencing 
programme adoption. For example, engagement activities designed to promote staff 'buy in' prior to programme adoption are recommended. It is possible that our findings are not generalisable, but they are consistent with those reported elsewhere [41-43].

Findings suggest that future programmes involving GP referral to physical activity should consider involving surgery staff in programme development, to identify physical activity champions to influence effective GP referral implementation when the programme is up-andrunning. In terms of physical activity session implementation, consistent with previous reviews $[13,16]$ the support and guidance provided by programme deliverers (i.e. exercise specialists) was seen to be central to the positive changes observed in respondent outcomes. Exercise specialists were credited with providing a catalyst for change from 'inactive' to 'active', and for recognising the importance of providing instructions on how to perform physical exercises; an approach advocated in a recent systematic review of physical activity interventions for inactive adults [44].

While previous reviews have identified benefits associated with group-based physical activity interventions in general [16], this is one of the first known studies to lend support to group-based interventions targeted specifically at inactive individuals living with a long-term condition. This finding is encouraging given evidence for positive associations between social support and physical activity participation among adults $[45,46]$ and older adults, [47].

The total cost of implementing CLICK into Activity over 3 years was approximately $£ 175,000$, with an average cost per person attending at least one session of $£ 535$. Unfortunately there is a lack of reporting on implementation costs and resources associated with adults' physical activity interventions, $[16,39,48]$ making it difficult to compare our findings with those of similar studies. However, the estimated cost of programme implementation compares favourably with the direct costs of disease management and common health conditions related to physical inactivity (not including costs to other parts of the NHS and wider health and social care system) [4].

\section{Strengths and limitations}

Strengths of the study include application of the REAIM framework, including collation and triangulation of qualitative and quantitative data from a variety of sources; overcoming challenges in the evaluation of physical activity programmes [22, 39, 49] and social prescribing schemes [41-43] that have been reported previously. Limitations of the study include a lack of control or comparison group. Secondly, we were unable to recruit individuals that never participated in a CLICK into
Activity session to participate in a qualitative interview; this limits our understanding of the barriers influencing initial engagement and participation. Thirdly, while our statistical approach was chosen precisely to mitigate the effects of missing data [29], 6- and 12-month follow-up response rates were low, which limits understanding of longer term programme effects. Fourthly, physical activity outcomes were based on self-report data collected by trained exercise specialists. Given reports of positive rapport developed between participants and exercise specialists, it is possible that participants over-reported activity levels for social approval, as observed in previous studies $[50,51]$. The collection of physiological data relating to participants' long-term conditions (for example, HbA1c levels among those participants with type 2 diabetes) was beyond the remit of this evaluation. In the case of type 2 diabetes, studies have shown that physical activity can improve glycaemic control among diabetic populations [52] and it could reduce type 2 diabetes incidence [53]. Future studies examining physiological as well as physical and mental health outcomes would be beneficial.

\section{Conclusions}

This study used the RE-AIM framework to evaluate the individual and organisational impacts of GP referral of inactive adults living with (or at risk of) long-term conditions to community-based physical activity. Although the target for programme reach was not met, and 6- and 12- month follow-up questionnaire responses were low, positive changes in physical activity and other outcomes assessed were observed among individuals that took part. Programme adoption at GP surgeries was successful, however, the GP referral process was not consistently implemented across participating surgeries. Physical activity sessions were successfully implemented; programme deliverers and group-based delivery were each identified as having an influential impact on programme outcomes, while changes to physical activity session content were made in response to participant feedback. An assessment of costs demonstrated the programme's potential value for money. Overall, findings highlight strategies to be explored in future development and implementation of GP referral to community-based physical activity programmes targeting inactive adults living with (or at risk of) long-term conditions.

\section{Supplementary information}

Supplementary information accompanies this paper at https://doi.org/10. 1186/s12889-019-7701-5.

Additional file 1. CLICK into Activity logic model. This table is a logic model, presenting the programme delivery plan and hypothesised outcomes. 
Additional file 2. Interview schedule for CLICK into Activity participants. This table presents an example interview schedule for CLICK into Activity participants.

Additional file 3. Consolidated criteria for reporting qualitative studies (COREQ). This table reports on the processes followed in undertaking the qualitative aspect of the research.

\section{Abbreviations}

BMI: Body mass index; COREQ: Consolidated criteria for reporting qualitative research; GP: General practice; IPAQ: International Physical Activity Questionnaire; NHS: National Health Service; RE-AIM: Reach, effectiveness, adoption, implementation, maintenance; WEMWBS: Warwick Edinburgh Mental Wellbeing Scale

\section{Acknowledgements}

We wish to thank members of the CLICK into Activity project steering group for their facilitation of this programme and for providing feedback on programme implementation. We would also like to acknowledge and thank all participants and stakeholders that supported this evaluation.

\section{Authors' contributions}

The study was conceived by ELB and JEP. ELB led data collection, developed the idea for the manuscript, analysed and interpreted the data, and wrote and edited the manuscript. JEP contributed to the conceptual content of the manuscript and the analysis of economic evaluation data. MSYB assisted with conducting participant interviews and initial coding of qualitative data. JEP and MSYB were involved in critically revising the paper for important intellectual content. All authors read and approved the final manuscript and agree to be accountable for the work.

\section{Authors' information}

ELB is a Senior Lecturer in Public Health at the University of the West of England, Bristol, UK. MSYB is a Research Associate in Public Health at the University of the West of England, Bristol, UK. JEP is Professor of Public Health Economics at the University of the West of England, Bristol, UK.

\section{Funding}

The authors would like to thank Sport England for funding this project through the Lottery supported Get Healthy, Get Active portfolio, with additional funding support from Somerset Health and Wellbeing Board, South Somerset District Council, the Broadway, Chard, Crewkerne and Ilminster (CLICK) Federation, and Somerset Activity and Sports Partnership. The funders had no role in study design, data collection and analysis, decision to publish, or preparation of the manuscript.

\section{Availability of data and materials}

This study does not have ethical approval to share the study dataset either in a repository or as supporting files. However, the data may be available from the corresponding author on reasonable request.

\section{Ethics approval and consent to participate}

This study was granted ethical approval by the University of the West of England Ethics Committee (Ref: HAS/15/08/008). All participants were provided with information about the evaluation and gave written informed consent to participate.

\section{Consent for publication}

Not applicable.

\section{Competing interests}

The authors declare that they have no competing interests.

Received: 5 December 2018 Accepted: 27 September 2019

Published online: 17 October 2019

\section{References}

1. Department of Health (UK). Start Active, Stay Active: A report on physical activity from the four home countries' Chief Medical Officers. 2011. https:// www.gov.uk/government/publications/start-active-stay-active-a-report-on- physical-activity-from-the-four-home-countries-chief-medical-officers. Accessed 6 Nov 2018

2. Lee I, Shiroma EJ, Lobelo F, Puska P, Blair SN, Katzmarzyk PT. For the lancet physical activity series working group. Impact of physical inactivity on the world's non-communicable diseases. Lancet. 2012;380(9838):219-29.

3. Health Survey for England Health Survey for England, 2016. 2017. https:// digital.nhs.uk/data-and-information/publications/statistical/health-survey-forengland/health-survey-for-england-2016. Accessed 6 Nov 2018.

4. Public Health England. Physical activity: applying all our health. 2018. https://www.gov.uk/government/publications/physical-activity-applying-allour-health/physical-activity-applying-all-our-health\#contents. Accessed 6 Nov 2018.

5. Everson-Hock ES, Green MA, Goyder EC, Copeland RJ, Till SH, Heller B, Hart O. Reducing the impact of physical inactivity: evidence to support the case for targeting people with chronic mental and physical conditions. J Public Health. 2016;38(2):343-51.

6. Public Health England. Everybody active, every day: an evidence-based approach to physical activity. 2014. https://www.gov.uk/government/ publications/everybody-active-every-day-a-framework-to-embed-physicalactivity-into-daily-life. Accessed 6 Nov 2018.

7. Sport England. Towards an active nation. 2016. https://www.sportengland. org/news-and-features/news/2016/may/19/sport-england-triplesinvestment-in-tackling-inactivity/. Accessed 6 Nov 2018.

8. Kimberlee R. What is social prescribing? Adv Soc Sci Res J. 2015;2(1):102-10.

9. Pescheny JV, Pappas Y, Randhawa G. Facilitators and barriers of implementing and delivering social prescribing services: a systematic review. BMC Health Serv Res. 2018;18:86.

10. Baumeister RF, Leary MR. The need to belong: desire for interpersonal attachments as a fundamental human motivation. Psychol Bull. 1995;117(3): 497-529.

11. Estabrooks PA, Harden SM, Burke SM. Group dynamics in physical activity promotion: what works? Soc Personal Psychol Compass. 2012:6(1):18-40.

12. Bickerdike $L$, Booth $A$, Wilson PM. Social prescribing: less rhetoric and more reality. A systematic review of the evidence. BMJ Open. 2017;7(4):e013384

13. Matthews L, Kirk A, MacMillan F, Mutrie N. Can physical activity interventions for adults with type 2 diabetes be translated into practice settings? A systematic review using the RE-AIM framework. TBM. 2014;4:60-78.

14. Gardner B, Smith L, Lorencatto F, Hamer M, Biddle SJ. How to reduce sitting time? A review of behaviour change strategies used in sedentary behaviour reduction interventions among adults. Health Psychol Rev. 2016;10(1):89112.

15. Estabrooks PA, Bradshaw M, Fox E, Berg J, Dzewaltowski DA. The relationships between delivery agents' physical activity level and the likelihood of implementing a physical activity program. Am J Health Promot. 2004;18(5):350-3.

16. Harden SM, McEwan D, Sylvester BD, Kaulius M, Ruissen G, Burke SM, Estabrookes PA, Beauchamp MR. Understanding for whom, under what conditions, and how group-based physical activity interventions are successful: a realist review. BMC Public Health. 2015:15:958.

17. Glasgow RE, Vogt TM, Boles SM. Evaluating the public health impact of health promotion interventions: the RE-AIM framework. Am J Public Health. 1999;89(9):1322-7.

18. Jauregui E, Pecheco AM, Soltero EG. Using the RE-AIM framework to evaluate physical activity public health programs in México. BMC Public Health. 2015;15:162.

19. NICE. Type 2 diabetes: prevention in people at high risk. Public Health guideline [PH38]. 2012. https://www.nice.org.uk/guidance/ph38. Accessed 6 Nov 2018.

20. Department of Health. Start Active Stay Active. 2011. https://www.gov.uk/ government/publications/start-active-stay-active-a-report-on-physicalactivity-from-the-four-home-countries-chief-medical-officers. Access $6 \mathrm{Nov}$ 2018

21. Adams EJ, Chalkley AE, Esliger DW, Sherar LB. Evaluation of the implementation of a whole-workplace walking programme using the REAIM framework. BMC Public Health. 2017;17:466.

22. Koorts H, Gillison F. Mixed method evaluation of a community-based physical activity program using the RE-AIM framework: practical application in a real-world setting. BMC Public Health. 2015:15:1102.

23. Craig C, Marshall A, Sjostrom M, Bauman A, Booth M, Ainsworth B. International physical activity questionnaire: 12-country reliability and validity. Med Sci Sports Exerc. 2003;35:1381-95. 
24. Stewart-Brown SL, Platt S, Tennant A, Maheswaran H, Parkinson J, Weich S, Tennant R, Taggart F, Clarke A. The Warwick-Edinburgh mental well-being scale (WEMWBS): a valid and reliable tool for measuring mental well-being in diverse populations and projects. JECH. 2011;65:2.

25. Leong DP, Teo KK, Rangarajan S. Prognostic value of grip strength: findings from the prospective urban rural epidemiology (PURE) study. Lancet. 2015; 386(9990):266-73.

26. Jago R, Edwards MJ, Sebire SJ, Tomkinson K, Bird EL, Banfield K, May T, Kesten JM, Cooper AR, Powell JE, Blair PS. Effect and cost of an after-school dance programme on the physical activity of 11-12 year old girls: the Bristol girls dance project school-based cluster randomised controlled trial. IJBNPA. 2015;12:128

27. Tibbitts B, Porter A, Sebire SJ, Metcalfe C, Bird EL, Powell J, Jago R. Action 3: 30: protocol for a cluster randomised feasibility study of a revised teaching assistant led extra-curricular physical activity intervention for 8 to 10 year olds. Pilot Feasibility Stud. 2017;3:69.

28. IPAQ Research committee. Guidelines for data processing and analysis of the International Physical Activity Questionnaire (IPAQ) - Short Form 2014 https://sites.google.com/site/theipaq/. Accessed 6 Nov 2018.

29. Seltman HJ. Mixed Models. In HJ. Seltman (Eds), Experimental Design and Analysis. Pittsburgh: Carnegie Mellon University; 2018. p. 358-78.

30. Braun V, Clarke V. Using thematic analysis in psychology. Qual Res Psych. 2006;3:77-101.

31. Summers Holtrop J, Rabin BA, Glasgow RE. Qualitative approaches to use of the RE-AIM framework: rationale and approach. BMC Health Serv Res. 2018; 18:177.

32. Fusch PI, Ness LR. Are we there yet? Data saturation in qualitative research. Qual Rep. 2015;20(9):1408-16.

33. Tong A, Sainsbury P, Craig J. Consolidated criteria for reporting qualitative research (COREQ): a 32-item checklist for interviews and focus groups. Int J Qual Health Care. 2007;19(6):349-57.

34. Creswell JW, Plano Clark VL, Gutmann M, Hanson W. Advanced mixed methods research designs. In: Tashakkori A, Teddlie C, editors. Handbook of mixed methods in social and behavioral research. Thousand Oaks: Sage; 2003. p. 209-40.

35. Raynor HA, Osterholt KM, Hart CN, Jelalian E, Viver P, Wing RR. Evaluation of active and passive recruitment methods used in randomised controlled trials targeting pediatric obesity. Int J Pediatr Obes. 2011:4:224-32.

36. Cooke $\mathrm{R}$, Jones $\mathrm{A}$. Recruiting adult participants to physical activity intervention studies using sport: a systematic review. BMJ Open Sport Exerc Med. 2017;3:e000231.

37. Allender S, Cowburn G, Foster C. Understanding participation in sport and physical activity among children and adults: a review of qualitative studies. Health Educ Res. 2006;6(1):826-35.

38. Chief Medical Officer. UK physical activity guidelines. 2011. https://www.gov. uk/government/publications/uk-physical-activity-guidelines. Accessed 6 Nov 2018.

39. Baba CT, Oliviera IM, Silva AEF, Vieria LM, Cerri NC, Florindo AA, Angelica G, Gomes $\mathrm{O}$. Evaluating the impact of a walking programme in a disadvantaged area: using the RE-AIM framework by mixed methods. BMC Public Health. 2017:17:709.

40. Blackford K, Jancey J, Lee AH, James A, Howat P, Waddell T. Effects of a home-based intervention on diet and physical activity behaviours for rural adults with or at risk of metabolic syndrome: a randomised controlled trial. IJBNPA. 2016:13:13

41. Polley M, Bertotti M, Kimberlee R, Pilkington K, Refsum C. A review of the evidence assessing impact of social prescribing on healthcare demand and cost implications. 2017. https://westminsterresearch.westminster.ac.uk/item/ q1455/a-review-of-the-evidence-assessing-impact-of-social-prescribing-onhealthcare-demand-and-cost-implications. Accessed 6 Nov 2018

42. Farenden C, Mitchell C, Feast S, Verdenicci S. Community navigation in Brighton \& Hove. Eval Soc Prescribing Pilot. 2015; http://www.bh-impetus. org/wp-content/uploads/2015/12/CN-Full-Evaluation-Nov-2015.pdf. Accessed 6 Nov 2018.

43. The Health Foundation. Shine 2014 final report social prescribing: Integrating GP and community assets for health. 2015. https://www.health. org.uk/sites/health/files/City\%20and\%20Hackney\%20CCG\%20final\%20report. pdf. Accessed 6 Nov 2018.

44. Howlett N, Trivedi D, Troop NA, Chater AM. Are physical activity interventions for healthy inactive adults effective in promoting behaviour change and maintenance, and which behaviour change techniques are effective? A systematic review and meta-analysis. TBM. 2018. https://doi.org/ 10.1093/tbm/iby010.

45. Ford ES, Ahluwalia IB, Galuska DA. Social relationships and cardiovascular disease risk factors: findings from the third national health and nutrition examination survey. Prev Med. 2000;30(2):83-92.

46. McNeill LH, Wyrwich KW, Brownson RC, Clark EM, Kreuter MW. Individual, social environmental, and physical environmental influences on physical activity among black and white adults: a structural equation analysis. Ann Behav Med. 2006:31(1):36-44

47. Smith GL, Banting L, Eime R, O'Sullivan G, van Uffelen JGZ. The association between social support and physical activity in older adults: a systematic review. IJBNPA. 2017;14:56

48. Abu-Omar K, Rutten A, Burlacu I, Schatzlein V, Messing S, Cuhrcke M. The cost-effectiveness of physical activity interventions: a systematic review of reviews. Prev Med Rep. 2017:8:72-8.

49. Hanson S, Jones A. Missed opportunities in the evaluation of public health interventions: a case study of physical activity programmes. BMC Public Health. 2017;17:674.

50. Adams SA, Matthews CE, Ebbeling CB, Moore CG, Cunningham JE, Fulton J, Hebert JR. The effect of social desirability and social approval on self-reports of physical activity. Am J Epidemiol. 2005;161:389-98.

51. Shephard R. Limits to the measurement of habitual physical activity by questionnaires. Br J Sports Med. 2003;37:197-206.

52. Festa A, Williams K, D'Agostino R. The insulin resistance atherosclerosis study. Diabetes. 2006;55:1114-20.

53. Laaksonen DE, Lindstrom J, Lakka TA, et al. Physical activity in the prevention of type 2 diabetes: the Finnish diabetes prevention study. Diabetes. 2005:54:158-65.

\section{Publisher's Note}

Springer Nature remains neutral with regard to jurisdictional claims in published maps and institutional affiliations.

Ready to submit your research? Choose BMC and benefit from:

- fast, convenient online submission

- thorough peer review by experienced researchers in your field

- rapid publication on acceptance

- support for research data, including large and complex data types

- gold Open Access which fosters wider collaboration and increased citations

- maximum visibility for your research: over $100 \mathrm{M}$ website views per year

At BMC, research is always in progress.

Learn more biomedcentral.com/submissions 